\title{
Survey of Various Protocols in Geographical Based Routing in Vehicular Adhoc Networks
}

\author{
Nupur Soni \\ BBD University \\ Lucknow, UP, INDIA
}

\author{
Shikha Tiwari \\ BBD University \\ Lucknow, UP, INDIA
}

\begin{abstract}
Vehicular Ad hoc Network (VANET), a subclass of mobile ad hoc networks (MANETs), is a promising approach for the intelligent transportation system (ITS). The design of routing protocols in VANETs is important and necessary issue for support the smart ITS. The key difference of VANET and MANET is the special mobility pattern and rapidly changeable topology. It is not effectively applied the existing routing protocols of MANETs into VANETs VANET (Vehicular Ad-hoc Network) is a new technology which has taken enormous attention in the recent years. Due to rapid topology changing and frequent disconnection makes it difficult to design an efficient routing protocol for routing data among vehicles, called V2V or vehicle to vehicle communication and vehicle to road side infrastructure, called V2I. The existing routing protocols for VANET are not efficient to meet every traffic scenarios. Thus design of an efficient routing protocol has taken significant attention. So, it is very necessary to identify the pros and cons of routing protocols which can be used for further improvement or development of any new routing protocol. The easiest way to classify the geographic routing protocolsis by type of routing (Unicast, Broadcast or Geocast). Other way to classify them is by the use that the protocol gives to the position information (Packet forwarding, Route Selection, Cluster formation, Formation of cells, Classify Forwarding Group, or RouteRequest Forwarding). For this survey we will use the type of routing classification and in each protocol we'll talk about how the protocol uses the geographic information.
\end{abstract}

Keywords : VANET,Unicast,GPSR,Greedy Routing,A-STAR

\section{INTRODUCTION}

Vehicular ad hoc network is a special form of MANET which is a vehicle to vehicle \& vehicle roadside wireless communicationnetwork. It is autonomous \& self-organizing wireless communication network, where nodes in VANET involve themselves as servers and/or clients for exchanging \& sharing information. The network architecture of VANET can be classified into three categories: pure cellular/WLAN, pure ad hoc, and hybrid [1]. Due to new technology it has taken huge attention from government, academy \& industry.

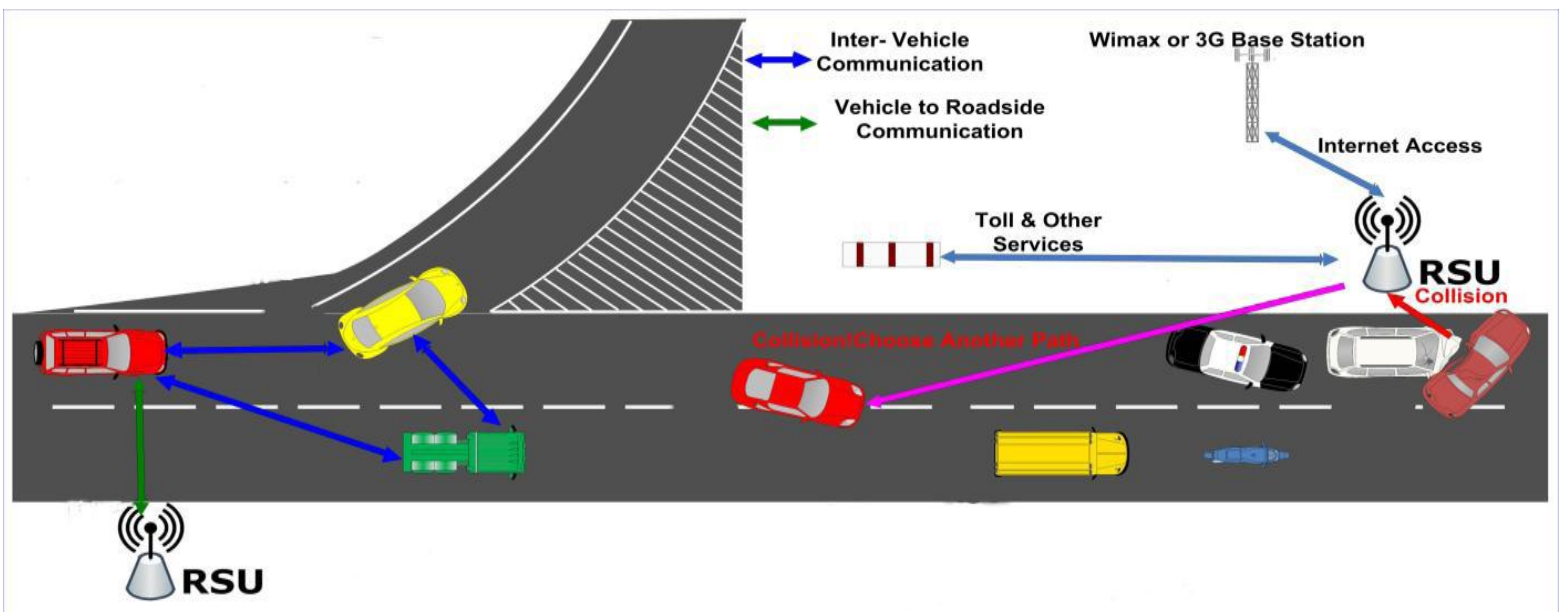

Figure-1[25]: shows a form of vehicular adhoc network. 
One of the outcomes has been a novel type of wireless access called Wireless Access for Vehicular Environment (WAVE) dedicated to vehicle-to-vehicle and vehicle-to-roadside communications. While the major objective has clearly been to improve the overall safety of vehicular traffic, promising traffic management solutions and onboard entertainment applications are also expected by the different bodies (C2CCC1, VII2, CALM3) and projects (VICS4 (Yamada, 1996), CarTALK 2000 (Reichardt D, 2002), NOW5, CarNet (Morris R, 2000), FleetNet (Franz, 2001)) involved in this field. When equipped with WAVE communication devices, cars and roadside units form a highly dynamic network called a Vehicular Ad Hoc Network (VANET), a special kind of Mobile Ad-Hoc Networks (MANETs). While safety applications mostly need local broadcast connectivity, it is expected that some emerging scenarios [2] developed for intelligent transportation systems (ITS) would benefit from unicast communication over a multi-hop connectivity. Moreover, it is conceivable that applications that deliver contents and disseminate useful information can flourish with the support of multihop connectivity in VANETs.

Although countless numbers of routing protocols [3][4] have been developed in MANETs, many do not apply well to VANETs. VANETs represent a particularly challenging class of MANETs. They are distributed, self-organizing communication networks formed by moving vehicles, and are thus characterized by very high node mobility and limited degrees of freedom in mobility patterns.

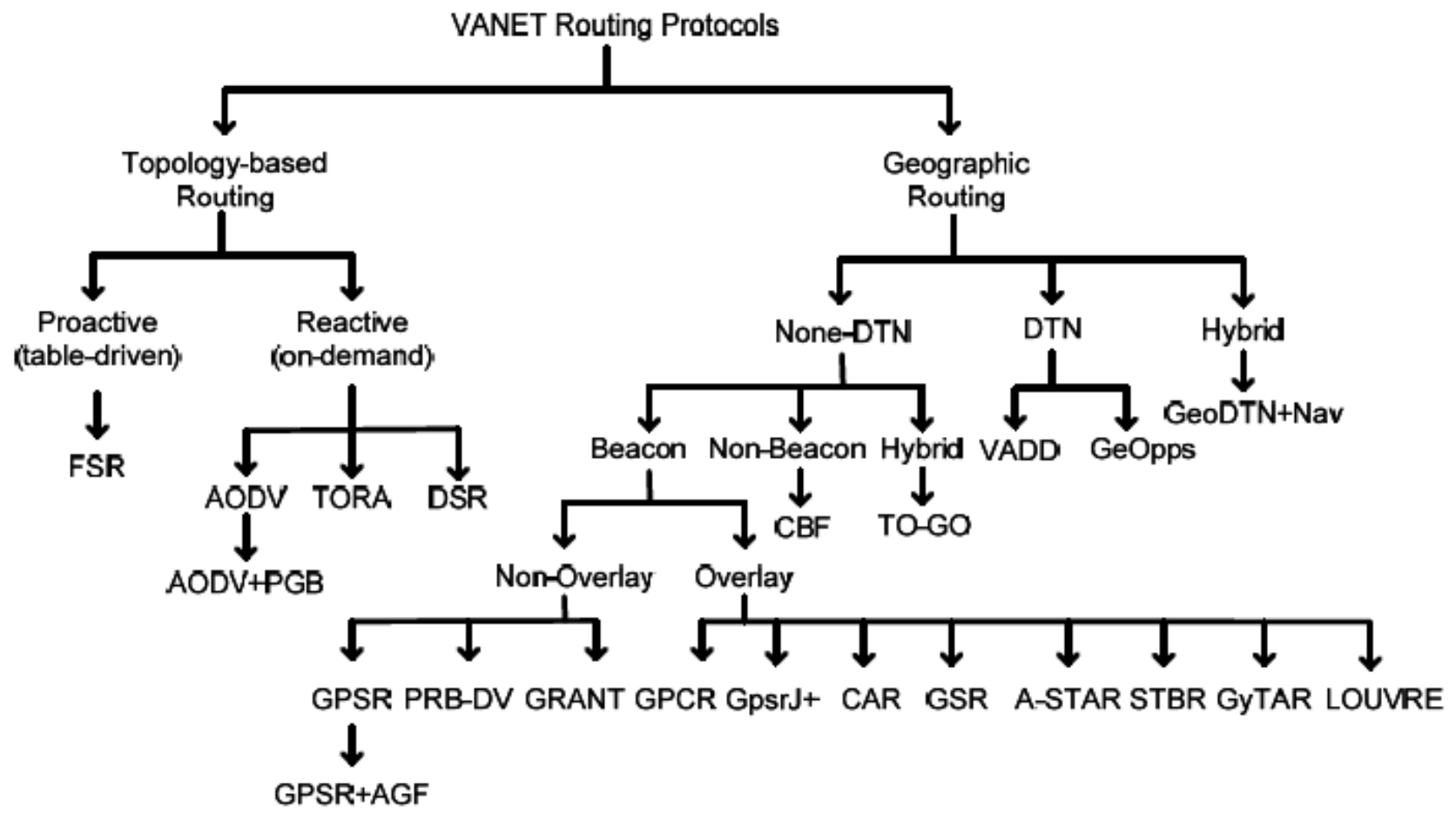

Figure 2[24]:Taxonomy of various Routing Protocols in VANET

As shown in Figure 2, there are two categories of routing protocols: topology-based and geographic routing. Topology-based routing uses the information about links that exist in the network to perform packet forwarding. Geographic routing uses neighboring location information to perform packet forwarding. Since link information changes in a regular basis, topology-based routing suffers from routing route breaks.

\section{GEOGRAPHIC (POSITION-BASED) ROUTING:}

In geographic (position-based) routing, the forwarding decision by a node is primarily made based on the position of a packet's destination and the position of the node's one-hop neighbors. The position of the destination is stored in the header of the packet by the source. The position of the node's one-hop neighbors is obtained by the beacons sent periodically with random jitter (to prevent collision). Nodes that are within a node's radio range will become neighbors of the node. Geographic routing assumes each node knows its location, and the sending node knows the receiving node's location by the increasing popularity of Global Position System (GPS) unit from an onboard Navigation System and the recent research on location services [5][6][7], respectively. Since geographic routing protocols do not exchange link state information and do not maintain established routes like proactive and reactive topology-based routings do, they are more robust and promising to the highly dynamicenvironments like VANETs. In other words, route is determined based on the geographic location of neighboring nodes as the packet is forwarded. There is no need of link state exchange nor route setup. Figure 1 subclassifies Geographic routing into three categories of non-Delay Tolerant Network (non-DTN), Delay Tolerant Network (DTN), and hybrid. The non-DTN types of geographic routing protocols do not consider intermittent connectivity and are only practical in densely populated VANETs whereas DTN types of geographic routing protocols do consider disconnectivity. However, they are designed 
from the perspective that networks are disconnected by default. Hybrid types of geographic routing protocols combine the non-DTN and DTN routing protocols to exploit partial network connectivity. We describe these three sub-categories in the following:

Non-DTN - Overlay The fundamental principle in the greedy approach is that a node forwards its packet to its neighbor that is closest to the destination. The forwarding strategy can fail if no neighbor is closer to the destination than the node itself. In this case, we say that the packet has reached the local maximum at the node since it has made the maximum local progress at the current node.
The routing protocols in this category have their own recovery strategy to deal with such a failure.

GPSR - In Greedy Perimeter Stateless Routing (GPSR) [8], a node forwards a packet to an immediate neighbor which is geographically closer to the destination node. This mode of forwarding is termed greedy mode. When a packet reaches a local maximum, a recovery mode is used to forward a packet to a node that is closer to the destination than the node where the packet encountered the local maximum. The packet resumes forwarding in greedy mode when it reaches a node whose distance to the destination is closer than the node at the local maximum to the destination.

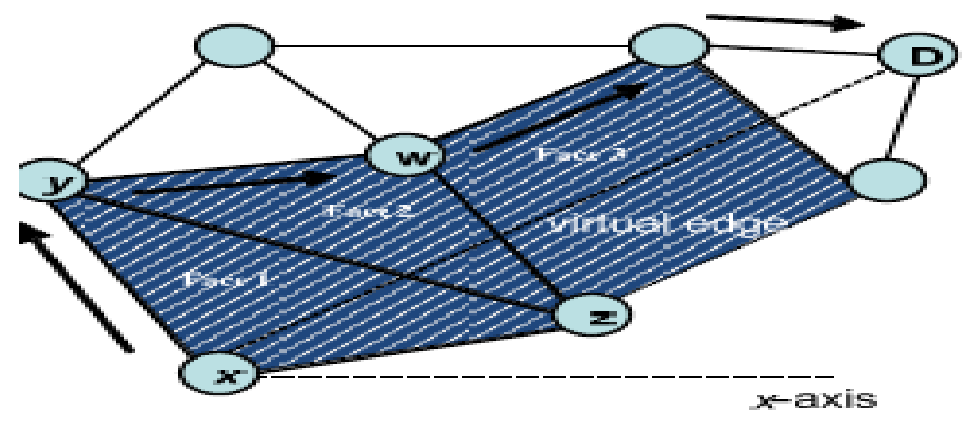

Figure 3[24]: Right-hand rule in GPSR's perimeter mode; packet performs face routing to route along Face 1, Face 2, and Face 3 toward destination D.

GPSR recovers from a local maximum using perimeter mode based on the right-hand rule shown in Figure 3. The rule states that when a node $x$ first enters into the recovery mode, its next forwarding hop $y$ is the node that is sequentially counterclockwise to the virtual edge formed by $x$ and destination $D$. Afterwards, the next hop $z$ is sequentially counterclockwise to the edge formed by $y$ and its previous node $x$ shown in Figure 3. While walking the face, however, if the edge $y z$ formed by the current node and the next hop crosses the virtual edge $x D$ and results in a point that is closer than the previous intersecting point $x$, perimeter mode will perform a face change in that the next hop $w$ is chosen sequentially counterclockwise to the edge $y z$ where the closer intersecting point was found. Such routing is called face routing because the packet traverses many faces formed by nodes in the network until it reaches a node closer to the destination than where the packet entered in the perimeter mode and where the face routing started
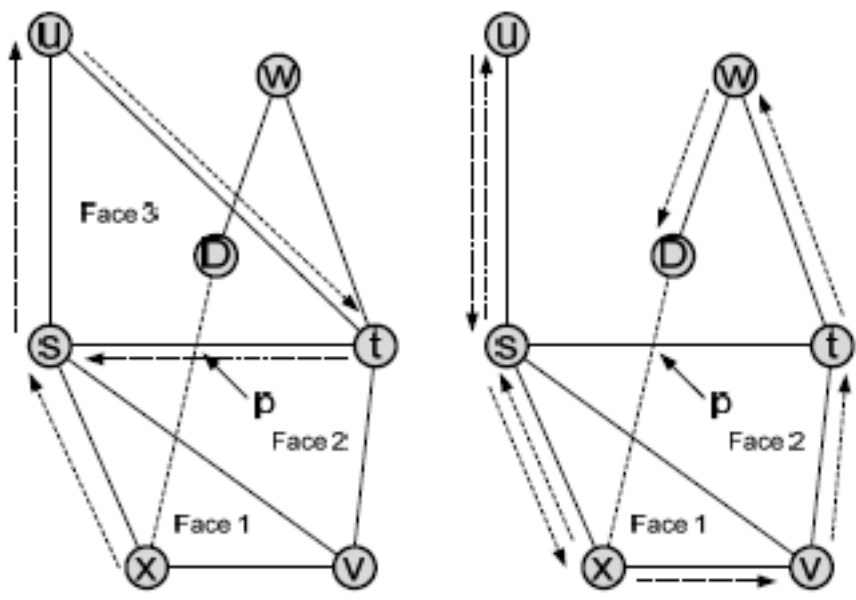

Figure 4[24]: On the left, packet will loop around face 3; on the right, packet will eventually route to D through u, s, x, v, t, and w. 
Note that if the graph is not planar, that is, there are cross edges in the graph, routing loops may occur. Consider Figure 4, $x$ tries to reach $D$ in perimeter mode. The packet will eventually loop around face 3 with no intersecting point closer than $p$. Had the cross edge $u t$ been removed, the packet would travel the exterior face $u, s, x, v, t$, and $w$ to reach $D$. Given that perimeter mode must operate on planar graphs to avoid routing loops, GPSR provided two distributed algorithms that produce Relative Neighborhood Graph (RNG) [9] and [10] which are known to be planar. Both RNG and GG algorithms yield a connected planar graph so long as the connectivity between two nodes obeys the unit graph assumption: for any two vertices, they must be connected by an edge if the distance between them is less than or equal to some threshold distance $d$ and must not be connected by an edge if the distance between them is greater than $d$. However, the unit graph assumption is not true in VANETs due to channel fading (obstacles and mobility). As a result, planar graphs are usually hard to achieve in VANETs.

GPSR+AGF - [11] observed two problems with GPSR in VANETs. First, due to the mobile nature of VANETs, a node's neighbor table often contains outdated information of neighbors' position. The problem can be solved by increasing beacons' frequency, yet such a solution only increases congestion and brings in potential collisions. The second problem is that the destination's location within the packet is never updated despite the destination is moving. To address these two problems, the authors proposed Advanced Greedy Forwarding (AGF) that incorporates the speed and direction of a node in the beacon packet and the total travel time, including the time to process the packet, up to the current forwarding node within the data packet. With the velocity vector, speed plus direction, each node can filter out outdated nodes in its neighbor table. With the total travel time, each forwarding node can better determine the deviation of the destination's original location and estimate its current location. Results have shown at least three times of improvement in packet delivery ratio to GPSR.

PRB-DV - Position-Based Routing with Distance Vector Recovery (PBR-DV) uses AODV-style recovery as packets fall into a local maximum. The node at the local maximum would broadcast a request packet in which is the node's position and destination's location. Upon receiving a request packet, a node would first check if it is closer to the destination than the node at the local maximum. If it is not, it records the node from which it receives the request packet (similar to backward learning) and rebroadcasts the request; otherwise, it sends a reply to the node from which it receives the request. As the reply packet travels back to the local maximum node, every intermediate node will record the previous node from which it receives the reply packet so that the local maximum node can maintain a route to a closer node than itself. The disadvantage of this scheme is that addition flooding is necessary to discover the nongreedy part of the route. There is no evaluation done comparing PRB-
DV to GPSR nor AODV thus performance in packet delivery and overhead is inconclusive.

GRANT - Greedy Routing with Abstract Neighbor Table (GRANT) [12] uses the concept of extended greedy routing where every node knows its $x$ hop neighborhood. This gives every node a far sighted vision of the best route to take to avoid local maximum. The metric in selecting the next forwarding neighbor $E$ is based on the multiplication of the distance between the node $N, x$ hop away from $E$ and the destination, the shortest path from $N$ to $E$, and the charge . Since the evaluation is done on static traces and the $x$-hop neighbors are assumed to be available, the beacon overhead and possible inaccuracy are not measured and well understood. In addition, although there are more paths that have smaller path length than traditional greedy routing on a normalized percentage basis, there is no absolute performance metric such as packet delivery ratio that can validate its true performance. per hop for multihop neighbors. The neighbor $E$ that offers the smallest such metric will be chosen to be the next hop. Because transmitting $x$-hop neighbors in the beacon is too much overhead, GRANT separates the plane into areas and includes only one representative neighbor per area. Upon receiving a beacon, a node computes the area that the broadcasting node and its neighbors belong to, thus categorizing them into different hops from the current node. The evaluation is based on snapshots of placement of cars from a uniform distribution.

\section{Overlay:}

An overlay routing has the characteristic that the routing protocol operates on a set of representative nodes overlaid on top of the existing network. In the urban environment, it is not hard to observe that decisions are made at junctions as these are the places where packets make turns onto a different road segment. Therefore, the overlaid routing protocols presented below have something to do with nodes at junction.

GPCR - A new routing approach for mobile Ad-Hoc Networks, called as Greedy Perimeter Coordinator Routing (GPCR), is introduced The main idea of GPCR is to take advantage of the fact that streets and junctions form a natural planar graph, without using any global or external information such as a static street map. GPCR consists of two parts: a restricted greedy forwarding procedure and a repair strategy which is based on the topology of real-world streets and junctions and hence does not require a graph planarization algorithm. Junctions are the only places where actual routing decision are taken. $\square$ Therefore packets should always be forwarded to a node on a junction rather than beeing forwarded accross a junction. $\square$ Node $\boldsymbol{u}$ would forward the packet beyond the junction to node $\mathbf{1} \boldsymbol{a}$ if regular greedy forwarding is used. $\square$ By forwarding the packet to node $\mathbf{2} \boldsymbol{a}$ an alternative path to the destination node can be found without getting stuck in a local optimum A coordinator broadcasts its role along with its position information. In a first step we assume that each node 
International Journal of Computer Applications Technology and Research

Volume 2- Issue 3, 357 - 366, 2013, ISSN: 2319-8656

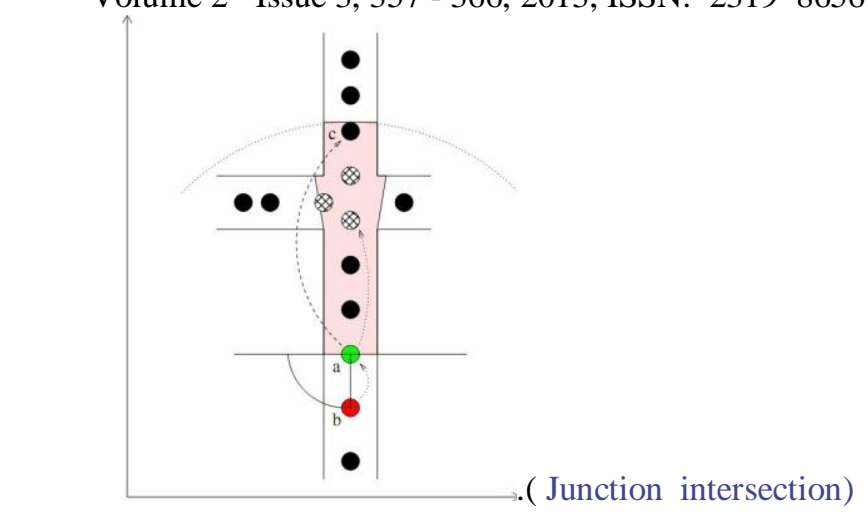

Figure 5[26]: Grredy Perimeter Coordinator Routing (GPCR)

knows whether it is a coordinator (i.e., located in the area of a junction) or not Figure 5 shows an example of how the next hop is selected on a street. $\square$ Node $\boldsymbol{a}$ receives a packet from node $\boldsymbol{b}$. Because $\boldsymbol{a}$ is located on a street and not on a junction it should forward the packet along this street. $\square$ First the qualified neighbors of a are determined. Then it is checked whether at least one of them is a coordinator. $\square$ As in this example there are three coordinator nodes thatqualify as a next hop one of these coordinator nodes is chosen randomly and the packet will be forwarded to this coordinator.

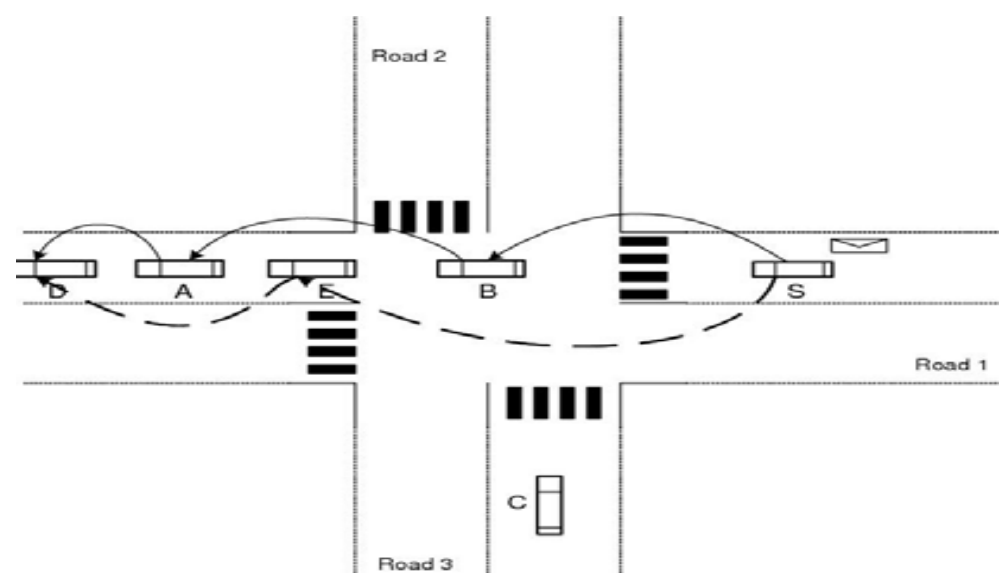

Figure 6[26]: Dashed arrows are GPSRJ+ and solid arrows are GPCR

GpsrJ+ - GpsrJ+ [13] removes the unnecessary stop at a junction while keeping the efficient planarity of topological maps. It uses twohop neighbor beaconing to predict which road segment its neighboring junction node will take. If the prediction indicates that its neighboring junction will forward the packet onto a road with a different direction, it forwards to the junction node; otherwise, it bypasses the junction and forwards the packet to its furthest neighboring node. Figure 6 illustrates the advantage of prediction. The figure shows that GpsrJ+ can bypass the junction area and forward the packet to node $E$ directly, yet GPCR forwards it to the junction node $B$, thus causing more transmissions. In the perimeter mode, GpsrJ+ uses the right-hand rule to determine the best direction (as opposed to final destination direction) and thereby the best forwarding node. That is, if the furthest node is in the same direction as the best direction, the best forwarding node is the furthest node; otherwise, the best forwarding node is a junction node. GpsrJ+ manages to increase packet delivery ratio of GPCR and reduces the number of hops in the recovery mode by $200 \%$ compared to GPSR.
CAR This work presents a novel position-based routing scheme called Connectivity-Aware Routing (CAR) $\square \square$ is designed specifically for inter-vehicle communication in a city and/or highway environment.CAR integrates locating destinations with finding connected paths

between source and destination. $\square$ "Guards" help to track the current position of a destination. The CAR protocol consists of four main parts:

1. Destination location and path discovery,

2. Data packet forwarding along the found path,

3. Path maintenance with the help of guards,

4. Error recovery.

\section{Adaptive beaconing}

The HELLO beacon includes location, moving direction and speed.

$\square$ The beaconing interval is changed according to the number of the 
registered nearby neighbors. $\square$ The fewer neighbors there are, the more frequent is a node's HELLO beaconing.
Therefore Node 3 in Figure 7 beacons more frequently than Nodes 2 and 4 and much more frequently than Node 1.

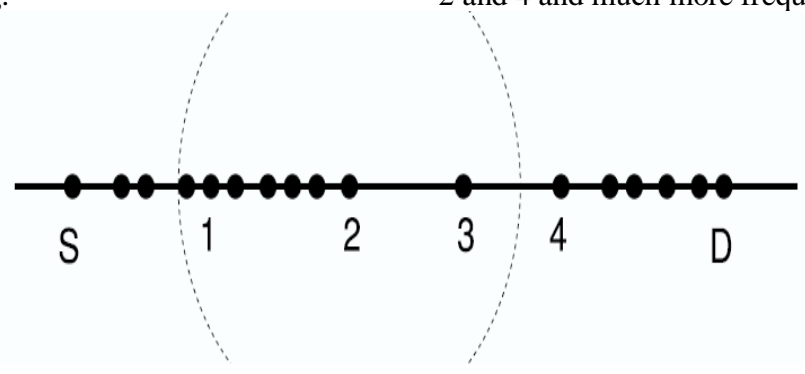

Figure. 7[26]: Influence of the neighbor table accuracy

The accuracy of node 1 neighbor table is far less important for the communication between nodes $S$ and $D$ than those of nodes 2,3 , and 4.

AGF is then used to forward the route reply back to the source via the recorded anchor points. When the source receives the route reply, it records the path to the destination and starts transmitting. Data packets are forwarded in a greedy manner toward the destination through the set of anchor points using AGF. In addition to handle mobility by AGF, CAR introduces "guards" to help to tack the current position of a destination. A guarding node can filter or redirect packets or adds information to a packet that will eventually deliver this information to the packet's destination.

The evaluation was done using a vehicular simulator and a probabilistic shadowing propagation model that uses a statistical approach to takes into account signal blockage. Results have shown CAR possesses higher packet delivery ratio (PDR) than GPSR and GPSR+AGF. The reason that CAR's PDR is higher than GPSR+AGF is that CAR guarantees to find the shortest connected path whereas GPSR+AGF may suffer from suboptimality of greedy mode in terms of finding such a path. CAR's path discovery overhead is checked by PGB. The overhead of storing guard is not in the data packets but in the beacons. According to their finding, a node on average only broadcasts 2-3 guards during the simulation. Thus, the beacon overhead is not overwhelming.

GSR - Geographic Source Routing (GSR) [14] relies on the availability of a map and computes a Dijkstra shortest path on the overlaid graph where the vertices are junction nodes and the edges are streets that connect those vertices. The sequence of junctions establishes the route to the destination. Packets are then forwarded greedily between junctions. GSR does not consider the connectivity between two junctions; therefore, the route might not be connected through. Recovery when such a case happens is greedy forwarding. The major difference between GSR and CAR is that CAR does not use a map and it uses proactive discovery of anchor points that indicate a turn at a junction.

As mentioned above, the movements of 955 vehicles are simulated by the traffic flow simulator Videlio [15], that incorporates a special lane changing model. The evaluation also considers a basic form of obstacle modeling as the propagation model. Simulation results have shown that GSR performs better than AODV and DSR in packet delivery ratio. In a densely populated network, most roads are connected that GSR forwards most of the packets. Scalability is not a problem to GSR as to AODV and DSR. However, GSR is not compared with other position-based routing protocols. Its performance in sparse networks is not verified.

\begin{abstract}
A-STAR-
Anchor-Based Street and Traffic Aware Routing [16] (A-STAR) is a position based routing protocol which is specially design for city scenarios for inter vehicle communication system. It ensures high connectivity in packet delivery by using vehicular traffic

city bus information for an end-to-end connection However, A-STAR is traffic aware: the traffic on the road determines whether the anchor points of the road will be considered in the shortest path. A-STAR routes based on two kinds of overlaid maps: a statically rated map and a dynamically rated map. A statistically rated map is a graph that displays bus routes that typically imply stable amount of traffic. Dijkstra paths computed over the statistically rated map are in general connected because of the extra knowledge. A dynamically rated map is a map that is generated based on the real-time traffic condition on the roads. Road-side deployment units can monitor the city traffic condition and distribute this information to every vehicle. Thus, the difference between a statically rated map and a dynamically rated map is accuracy of road traffic; while a statically rated map is based on bus routes that typically have high traffic volume, a dynamically rated map is based on the traffic monitored dynamically by road-side units. The mobility model and propagation model are based on the MGrid mobility model, a variant of the Manhattan model that considers not only the vehicular movement in a typical metropolis where streets are set out on a grid pattern but also the radio obstacles. A-STAR is compared to GSR and GPSR. Its packet delivery ratio is lower than GSR and GPSR with or without recovery as A-STAR can select paths with higher connectivity.
\end{abstract}

Street Topology-Based Routing(STBR) [17] is based on the ideaOf elucidate a given street map as a planar graph which has three valid states: master, slave, and forwarder for a node. In STBR one node is selected as a master on a junction, other nodes act as slaves \& intermediate nodes between junctions act as forwarders In STBR, packets are routed based on their geographic distance to the street where the destination is on. This is different from GSR or ASTAR where routes are computed through Dijkstra shortest path.

GyTAR - Greedy Traffic Aware Routing protocol (GyTAR) [18] is an overlaid approach similar to the approaches mentioned above in that packets are forwarded greedily toward the next junction which will then determine the best junction to forward next. GyTAR assumes that the number of cars is given per each road from roadside units and determines the connectivity of roads. A score is given to each neighboring junction considering the traffic density and their distance to the destination. The weights to traffic density and their distance to the destination are configurable parameters. GyTAR tries 
International Journal of Computer Applications Technology and Research

Volume 2- Issue 3, 357 - 366, 2013, ISSN: 2319-8656

to mimic the shortest path routing by taking into account the road connectivity. Simulations are based on a $2500 \mathrm{~m}$ x $2000 \mathrm{~m}$ map of 100 to 300 nodes. The movement of cars is adapted to the mobility model from (Davis, et al., 2001). GSR is compared to GyTAR which shows better packet delivery ratio. However, since it is not compared to any other overlaid routing protocol in this category, it is hard to gauge its relative performance.

LOUVRE - [13] has summarized geographic greedy overlay routing into two camps. The first camp is geo-reactive overlay routing where the next overlaid node is determined based on their neighboring nodes' distance to the destination (STBR) or a combination of it and traffic density (GyTAR). The second camp is geo-proactive overlay routing where the sequence of overlaid nodes is determined a-priori (GSR and A-STAR). Landmark Overlays for Urban Vehicular Routing Environments (LOUVRE) belongs to the second camp. It takes note of the fact that above a given vehicular density threshold, an overlay link remains connected regardless of the vehicular spatio-temporal distribution on the link. Thus, by only considering overlay links based on such density threshold when establishing overlay routes, most routes would partially use the same overlay links. With these considerations, geo-proactive overlay routing becomes attractive as it guarantees global route optimality and reduces the delay for establishing overlay routes. The drawback of this approach is obviously its scalability.

CBF: Contention-Based Forwarding (CBF) [19] is a geographic routing protocol that does not require proactive transmission of beacon messages. Data packets are broadcast to all direct neighbors and the neighbors decide if they should forward the packet. The actual forwarder is selected by a distributed timer-based contention process which allows the most-suitable node to forward the packet and to suppress other potential forwarders. Receivers of the broadcast data would compare their distance to the destination to the last hop's distance to the destination. The bigger the difference, the larger is the progress and shorter is the timer.

CBF is compared with GPSR with the perimeter mode disabled and with beacons of different intervals using realistic movement patterns of vehicles on a highway. With beacon interval of 0.25 seconds (the lowest set in the experiment), the packet delivery ratio (PDR) of GPSR is still not as good as that of CBF. As the beacon interval increases (up to 2 seconds), its PDR drops. (Please revise) Evaluation also shows that as the communication distance and thus the number of

hops a packet has to travel increases, the load on the wireless medium increases more for GPSR than CBF due to GPSR's constant beaconing overhead.

Hybrid: TOpology-assist Geo-Opportunistic Routing (TO-GO) [20] is a geographic routing protocol that exploits topology knowledge acquired via 2-hop beaconing to select the best target forwarder and incorporates opportunistic forwarding with the best chance to reach it. It is different from $\mathrm{CBF}$ in three main aspects. First, rather than picking the next forwarding node that makes the best progress to the destination, it picks the next forwarding node that makes the best progress to a target node. A target node is defined to be the node that greedy algorithm or recovery algorithm would normally pick except at the junction where optimization in choosing the target node either beyond the junction or at the junction is based upon whether the routing is in greedy mode or recovery mode. The reason for choosing the target node instead of the destination as the frame of reference is to take care of the city topology where roads intersect and destination usually does not lie on the same street as the source as in the highway. Packets have to make multiple turns into different streets before arriving at the destination. The data is then broadcast to all direct neighbors. Whoever's distance is closer to the target node gets picked to be the next forwarding node.

DTN: Delay Tolerant Network (DTN) uses carry \& forward strategy to overcome frequent disconnection of nodes in the network. In carry \& forward strategy when a node can't contact with other nodes it stores the packet \& forwarding is done based on some metric of nodes neighbors.). Since nodes are highly mobile, in this type of a network, they suffer from frequent disconnections. To overcome this, packet delivery is augmented by allowing nodes to store the packets when there is no contact with other nodes, to carry the packets for some distance until meeting with other nodes, and to forward based on some metric on nodes' neighbors (called carry-andforward strategy). The notable DTN vehicular routing protocols are VADD and GeOpps described below.

VADD - Vehicle-Assisted Data Delivery (VADD) [21] is a vehicular routing strategy aimed at improving routing in disconnected vehicular networks by the idea of carry-and-forward based on the use of predictable vehicle mobility. A vehicle makes a decision at a junction and selects the next forwarding path with the smallest packet delivery delay. A path is simply a branched road from an intersection. The expected packet delivery delay of a path can be modeled and expressed by parameters such as road density, average vehicle velocity, and the road distance. The minimum delay can be solved by a set of linear system equations. Zhao et. al. have introduced variations of VADD that chooses the next forwarding node after the next forwarding path has been determined. Location First Probe (LVADD) would select a node closest to the next forwarding path even though such a node is going away from the forwarding path. Direction First Probe (D-VADD) would select a node which is going toward the forwarding path even though such a node might be further from the forwarding path than other nodes on the path. Multi-Path Direction First Probe (MD-VADD) would select multiple nodes going toward the forwarding path so as not to miss forwarding to a node that offers a shorter time to the destination. Finally, Hybrid Probe (H-VADD) combines L-VADD and D-VADD so the long packet delay from D-VADD is offset by L-VADD and routing loops from L-VADD are masked by D-VADD. Results comparing with GPSR plus buffer and various versions of VADD show that $\mathrm{H}$ $\mathrm{VADD}$ has the best performance.

GeOpps - Geographical Opportunistic Routing (GeOpps) [23] takes advantage of the suggested routes of vehicles' navigation system to select vehicles that are likely to move closer to the final destination of a packet. It calculates the shortest distance from packet's destination to the nearest point (NP) of vehicles' path, and estimates the arrival of time of a packet to destination. Figure 9 shows Node $A$ in computing the NP of its neighbors N1 and N2. Since N2 offers closer NP to the destination, Node $A$ picks $N 1$ to forward its packets.

During the travel of vehicles, if there is another vehicle that has a shorter estimated arrival time, the packet will be forwarded to that vehicle. The process repeats until the packet reaches destination. The minimum delay used by VADD is indirectly obtained by selecting the next forwarding node whose path's nearest point is closest to the destination. GeOpps requires navigation information to be exposed to the network, thus, privacy such as vehicle's whereabouts might be an issue. 
International Journal of Computer Applications Technology and Research

Volume 2- Issue 3, 357 - 366, 2013, ISSN: 2319-8656

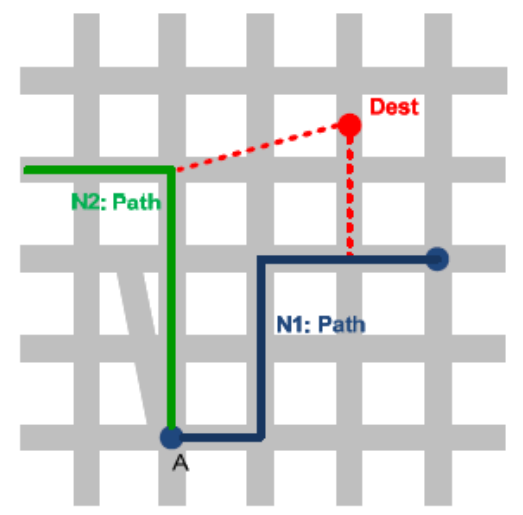

Figure 8[24]: Calculation of the Nearest Point (NP) from packet's Destination (D) for N1 and N2

Hybrid: GeoDTN+Nav [22] is a hybrid of non-DTN and DTN approach that includes the greedy mode, the perimeter mode, and the DTN mode. It switches from non-DTN mode to DTN mode by estimating the connectivity of the network based on the number of hops a packet has travelled so far, neighbor's delivery quality, and neighbor's direction with respect to the destination.. In addition to its hybrid approach, VNI offers users the option to protect their private data and at the same time provides best-effort routing decision.

Table 1. Summary of VANET TOPOLOGICAL routing protocols

\begin{tabular}{|c|c|c|c|c|c|}
\hline Routing Protocol & Type & Sub-Types & Overhead & $\begin{array}{l}\text { Mobility } \\
\text { Model }\end{array}$ & $\begin{array}{l}\text { Propagation } \\
\text { Model }\end{array}$ \\
\hline GPSR & Position-based & $\begin{array}{l}\text { Non-DTN, } \\
\text { Non-Overlay }\end{array}$ & Beacons & MTS & $\begin{array}{l}\text { Probabilistic } \\
\text { shadowing }\end{array}$ \\
\hline GPSR+AGF & Position-based & $\begin{array}{l}\text { Non-DTN, } \\
\text { Non-Overlay }\end{array}$ & Beacons & MTS & $\begin{array}{l}\text { Probabilistic } \\
\text { shadowing }\end{array}$ \\
\hline PRB-DV & Position-based & $\begin{array}{l}\text { Non-DTN, } \\
\text { Non-Overlay }\end{array}$ & $\begin{array}{l}\text { Beacons and } \\
\text { path states }\end{array}$ & Unknown & Unknown \\
\hline GRANT & Position-based & $\begin{array}{l}\text { Non-DTN, } \\
\text { Non-Overlay }\end{array}$ & $\begin{array}{l}\text { Two-hop } \\
\text { beacons }\end{array}$ & $\begin{array}{l}\text { Static trace } \\
\text { from a uniform } \\
\text { distribution }\end{array}$ & Road blocking \\
\hline GPCR & Position-based & $\begin{array}{l}\text { Non-DTN, } \\
\text { Non-Overlay }\end{array}$ & Beacons & VanetMobisim & Road blocking \\
\hline GpsrJ+ & Position-based & $\begin{array}{l}\text { Non-DTN, } \\
\text { Overlay }\end{array}$ & Beacons & VanetMobisim & Road blocking \\
\hline$\overline{\text { CAR }}$ & Position-based & $\begin{array}{l}\text { Non-DTN, } \\
\text { Overlay }\end{array}$ & $\begin{array}{l}\text { Path states and } \\
\text { beacons }\end{array}$ & MTS & $\begin{array}{l}\text { Probabilistic } \\
\text { shadowing }\end{array}$ \\
\hline GSR & Position-based & $\begin{array}{l}\text { Non-DTN, } \\
\text { Overlay }\end{array}$ & Beacons & $\begin{array}{l}\text { Videlio, } \\
\text { M-Grid moblity }\end{array}$ & Road blocking \\
\hline A-STAR & Position-based & $\begin{array}{l}\text { Non-DTN, } \\
\text { Overlay } \\
\end{array}$ & Beacons & $\begin{array}{l}\text { M-Grid } \\
\text { mobility }\end{array}$ & Road blocking \\
\hline STBR & Position-based & $\begin{array}{l}\text { Non-DTN, } \\
\text { Overlay }\end{array}$ & Beacons & Unknown & Unknown \\
\hline GyTAR & Position-based & $\begin{array}{l}\text { Non-DTN, } \\
\text { Overlay }\end{array}$ & Beacons & Proprietory & Free space \\
\hline LOUVRE & Position-based & $\begin{array}{l}\text { Non-DTN, } \\
\text { Overlay }\end{array}$ & Beacons & VanetMobisim & Road blocking \\
\hline $\mathrm{CBF}$ & Position-based & $\begin{array}{l}\text { Non-DTN, } \\
\text { Non-Beacon }\end{array}$ & Data boradcast & $\begin{array}{l}\text { Random way } \\
\text { point }\end{array}$ & $\begin{array}{l}\text { Two-Ray } \\
\text { ground } \\
\text { propagation } \\
\text { model }\end{array}$ \\
\hline
\end{tabular}


International Journal of Computer Applications Technology and Research

Volume 2- Issue 3, 357 - 366, 2013, ISSN: 2319-8656

\begin{tabular}{|l|l|l|l|l|l|}
\hline TO-GO & Position-based & $\begin{array}{l}\text { Non-DTN, } \\
\text { Hybrid }\end{array}$ & $\begin{array}{l}\text { Beacons and } \\
\text { data broadcast }\end{array}$ & VanetMobisim & Road blocking \\
\hline VADD & Position-based & DTN & Beacons & Unknown & Unknown \\
\hline GeOpps & Position-based & DTN & Beacons & MTS & None \\
\hline GeoDTN+Nav & Position-based & Hybrid & Beacons & VanetMobisim & Road blocking \\
\hline
\end{tabular}

\section{CONCLUSION}

This paper discusses various routing protocols of VANET. Designing an efficient routing protocol for all VANET applications is very difficult. Hence a survey of different VANET protocols, comparing the various features is absolutely essential to come up with new proposals for VANET. The performance of VANET routing protocols depend on various parameters like mobility model, driving environment and many more. Thus this paper has come up with an exhaustive survey and

\section{REFERENCES}

[1] Kevin C. Lee, Uichin Lee, Mario Gerla,"Survey of Routing Protocols in Vehicular Ad Hoc Networks," Advances in Vehicular Ad-Hoc Networks: Developments and Challenges, IGI Global, Oct, 2009

[2] Lee, K.C.; Lee, U.; Gerla, M. (2009), “TO-GO: TOpology-assist geo-opportunistic routing in urban vehicular grids," Wireless OnDemand Network Systems and Services, 2009. WONS 2009. ] Sixth International Conference on , vol., no., pp.11-18, 2-4 Feb. 2009..

[3] Mauve, et al. (2001), "A survey on position-based routing in mobile ad hoc networks," in IEEE Network Magazine, November/December 2001, pp. 30-39.

[4] Mehran A. et al. (2004), "A review of routing protocols for mobile ad hoc networks," in Ad Hoc Networks, Vol.2 pp.1-22, 2004.

[5] Flury, R. and Wattenhofer, R. (2006), "MLS: an efficient location service for mobile ad hoc networks." In MobiHoc '06: Proceedings of the 7th ACM international symposium on Mobile ad hoc networking and computing, pages 226-237, New York, NY, USA, 2006

[6] Li, J., Jannotti, J., D. S. J. D. Couto, D. R. Karger, and R. Morris (2000) "A scalable location service for geographic ad hoc routing." ], In MobiCom '00: Proceedings of the 6th annual international conference on Mobile computing and networking, pages 120-130, New York, NY, USA, 2000 comparison of different classes of VANET routing protocols. From the survey it is clear that position based, geocast and cluster based protocols are more reliable for most of the applications in VANET. In summary, the open issue in VANET routing is then whether there is any benchmark tool for evaluating these protocols. The research direction is that as VANET routings are advancing and becoming mature, many of the underlying assumptions and technologies will need to become mature as well so that much validity can be given to the benefits of these routing protocols.

[7] Y., Lu, G.-H., and Z.-L. Zhang (2004),"Enhancing location service scalability with highgrade. Mobile Ad-hoc and Sensor Systems," Yu. 2004 IEEE International Conference on, pages 164-173, 25-27 Oct. 2004.

[8] Karp, B. and Kung, H. T (2000), "GPSR: greedy perimeter stateless routing for wireless networks.”, In Mobile Computing and Networking, pages 243-254, 2000

[9] Toussaint, G. (1980), "The relative neighborhood graph of a finite planar set." Pattern Recognition, 12:231-268, 1980. Pattern Recognition, 12:231-268, 1980.

[10] Gabriel, K. R. and Sokal, R (1969), "A new statistical approach to geographic variation analysis." 18 Systematic Zoology, pages 231-268, 1969.

[11] Naumov, V., Gross, T.R. (2007), "Connectivity-Aware Routing (CAR) in Vehicular Ad-hoc Networks," INFOCOM 2007. 26th IEEE International Conference on Computer Communications. IEEE , vol., no., pp.1919-1927, 6-12 May, 2007.

[12] Schnaufer, S., Effelsberg, W. (2008), "Position-based unicast routing for city scenarios," World of Wireless, Mobile and Multimedia Networks, 2008. WoWMoM 2008. 2008 International Symposium on a , vol., no., pp.1-8, 23-26 June 2008.

[13] Lee, K. C., Haerri, J., Lee, U., and Gerla, M. (2007 )“Enhanced perimeter routing for geographic forwarding protocols in urban vehicular scenarios,"), Globecom Workshops, 2007 IEEE, pp. 1-10, 26-30 Nov. 2007. 
[14] C., Hartenstein, H., Tian, J., Fussler, H., Hermann, D., Mauve, M. (2003) ,"A routing strategy for vehicular ad hoc networks in city environments," Lochert, Intelligent Vehicles Symposium, 2003. Proceedings. IEEE, vol., no., pp. 156-161, 9-11 June 2003.

[15] Kronjäger, W. and Hermann D (1999) "Travel time estimation on the base of microscopic traffic flow simulation.", ITS World Congress, 1999.

[16] Seet, B.-C., Liu, G., Lee, B.-S., Foh, C. H., Wong, K. J.,Lee, K.K. (2004), "A-STAR: A Mobile Ad Hoc Routing Strategy for Metropolis Vehicular Communications."NETWORKING 2004, 989-999 [17] Forderer, D (2005). "Street-Topology Based Routing."

[17] Master's thesis, University of Mannheim, May 2005.

[18] Jerbi, M., Senouci, S.-M., Meraihi, R., and Ghamri-Doudane, Y. (2007), "An improved vehicular ad hoc routing protocol for city environments," Communications, 2007. ICC '07. IEEE International Conference, pp. 3972-3979, 24-28 June 2007.

[19] F“ußler, H., Hannes, H., J“org, W., Martin, M., Wolfgang, E. (2004), "Contention-Based Forwarding for Street Scenarios," Proceedings of the 1st International Workshop in Intelligent Transportation (WIT 2004), pages 155-160, Hamburg, Germany, March 2004

[20] Lee U., Cheung R., Gerla, M., "Emerging Vehicular Applications," Chapter 9 of Vehicular Networks: From Theory to Practice, Chapman \& Hall/Crc Computer and Information Science Series, March 17, 2009.

[21] Zhao, J.; Cao, G. (2006), "VADD: Vehicle-Assisted Data Delivery in Vehicular Ad Hoc Networks," INFOCOM 2006. 25th IEEE International Conference on Computer Communications. Proceedings, vol., no., pp.1-12, April 2006.

[22] Leontiadis, I., Mascolo, C. (2007), “GeOpps: Geographical Opportunistic Routing for Vehicular Networks," World of Wireless, Mobile and Multimedia Networks, 2007. WoWMoM 2007. IEEE International Symposium on a, vol., no., pp.1-6, 1821 June 2007.

[23] Cheng, P.-C., Weng, J.-T., Tung, L.-C., Lee, K. C., Gerla M., and Härri J. (2008), "GeoDTN+NAV: A Hybrid Geographic and DTN Routing with Navigation Assistance in Urban Vehicular Networks," Proceedings of the 1st International Symposium on Vehicular Computing Systems (ISVCS'08), Dublin, Irland, July 2008.

[24] Kevin C. Lee, Uichin Lee, Mario Gerla, "Survey of Routing Protocols in Vehicular Ad Hoc Networks"

[25] Bijan Paul,Md. Ibrahim,Md. Abu Naser Bikas, "VANET Routing Protocols: Pros and Cons".

[26] Valery Naumov, and Thomas R. Gross Proceedings of IEEE $26^{\text {th }}$ International Conference on Computer Communications

www.ijcat.com
(INFOCOM 2007), pp. 1919-1927, Anchorage, Alaska, USA, May 2007. 